Second Meeting, 11 th December 1896.

This meeting was postponed as the funeral of the President, Rev. John Wilson, M.A., F.R.S.E., took place that day.

Third Meeting, January 8th, 1897.

J. B. Clark, Esq., M.A., F.R.S.E., Vice-President, in the Chair.

\title{
Theorems on Normals of an Fillipse.
}

By Professor A. H. Avglin.

The condition that the normals at the points whose eccentric angles are $\alpha, \beta, \gamma$ shall be concurrent is

$$
\sin (\beta+\gamma)+\sin (\gamma+a)+\sin (a+\beta)=0 .
$$

The following method of establishing this result, as compared with those given in works on Concis, is direct, and also has the advantage of simplicity, by first proving the Trigonometrical identity

$$
\sin 2 \alpha \sin (\beta-\gamma)+\ldots=4 \sin \frac{\beta-\gamma}{2} \ldots\{\sin (\beta+\gamma)+\ldots\}, .
$$

which may be simply done by multiplying both sides of the wellknown identity

$$
\begin{gathered}
\sin (\beta-\gamma)+\sin (\gamma-\alpha)+\sin (\alpha-\beta) \\
=-4 \sin \frac{\beta-\gamma}{2} \sin \frac{\gamma-a}{2} \sin \frac{\alpha-\beta}{2} \\
\sin (\beta+\gamma)+\sin (\gamma+\alpha)+\sin (a+\beta),
\end{gathered}
$$

by

when it will be easily found that the product in the first nember reduces to that in (A) with opposite sign.

Now, writing the equation to the normal at $\phi$ in the form

$$
a x \sin \phi-b y \cos \phi=\frac{c^{2}}{2} \sin 2 \phi
$$


the normals at $\alpha, \beta, \gamma$ are concurrent if (eliminating $x, y$ as usual)

that is, if

$$
\left|\begin{array}{ccc}
\sin 2 a, & \sin a, & \cos a \\
\cdot & \cdot & \cdot \\
\cdot & . & \cdot
\end{array}\right|=0
$$

which, as shown above, is equivalent to

$$
\sin (\beta+\gamma)+\sin (\gamma+\alpha)+\sin (a+\beta)=0 .
$$

2. The normals at the angular points of a maximum triangle in (and so at the points of contact of a minimum triangle about) an ellipse are concurrent.

This may be shown without reference to the above reduced condition for concurrent normals.

For the condition

$$
\sin 2 a \sin (\beta-\gamma)+\ldots=0
$$

for concurrent normals follows at once from their equations; and at the angular points of a maximum inscribed triangle this condition is satisfied, for at these points we have

$$
\begin{gathered}
\beta-\alpha=\gamma-\beta=\frac{2 \pi}{3} ; \\
\therefore \quad \gamma-\alpha=\frac{4 \pi}{3}, \text { and } \gamma+\alpha=2 \beta .
\end{gathered}
$$

Hence

$$
\begin{gathered}
\sin 2 a \sin (\beta-\gamma)+\ldots \\
=-\sin \frac{\pi}{3}(\sin 2 \alpha+\sin 2 \beta+\sin 2 \gamma) \\
=-\sin \frac{\pi}{3}(\sin 2 \beta-\sin \overline{\gamma+\alpha})=0,
\end{gathered}
$$

and thus the normals are concurrent.

3. To find the area of the triangle formed by the three normals to an ellipse. 
In the case of the triangle formed by three lines whose equations are of the form $a x+b y+c=0$,

$$
\begin{aligned}
2 \Delta & =\left(a_{1} b_{2} c_{3}\right)^{2} / \Pi\left(a_{1} b_{2}\right) \\
& =\left[c_{1}\left(a_{2} b_{3}-a_{3} b_{2}\right)+\ldots\right]^{9} /\left(a_{2} b_{3}-a_{3} b_{2}\right) \ldots
\end{aligned}
$$

Hence, for the normals whose equations are of the form

we have

$$
a x \sin \phi-b y \cos \phi=\frac{c^{2}}{2} \sin 2 \phi,
$$

$$
\begin{aligned}
2 \Delta & =\left[\frac{c^{2} a b}{2}\{\sin 2 a \sin (\beta-\gamma)+\ldots\}\right]^{2} / a^{3} b^{3} \sin (\beta-\gamma) \ldots \\
& =\frac{c^{4}}{4 a b}\{\sin 2 a \sin (\beta-\gamma)+\ldots\}^{2} / \sin (\beta-\gamma) \ldots
\end{aligned}
$$

Now use the identity $(A)$, and we get

$$
2 \Delta=\frac{c^{4}}{2 a b} \tan \frac{\beta-\gamma}{2} \ldots\{\sin (\beta+\gamma)+\ldots\}^{2},
$$

the required expression.

4. If the normal at the points whose eccontric angles are $\alpha, \beta, \lambda, \delta$ be concurrent, then

$$
a+\beta+\gamma+\delta=(2 n+1) \pi .
$$

This is merely a question in Plane Trigonometry. For the equation to the normal at $\phi$ is

$$
a x \sec \phi-b y \operatorname{cosec} \phi=c^{2} \text {, }
$$

which is of the form asec $\phi-b \operatorname{cosec} \phi=c$.

Denoting $\tan \phi$ by $t$, we have

$$
\begin{aligned}
& a \sqrt{1+t^{2}}-\frac{b}{t} \sqrt{1+t^{2}}=c \\
\therefore \quad & \left(1+t^{2}\right)(a t-b)^{2}=c^{2} t^{2},
\end{aligned}
$$

an equation of the fourth degree in $t$, the roots of which are the tans. of the angles $a, \beta, \gamma, \delta$. And since the coefficients of $t$ and $t^{3}$ are obviously the same, we have, with the usual notation,

$$
\begin{gathered}
\tan (\alpha+\rho+\gamma+\delta) \equiv\left(s_{1}-s_{3}\right) /\left(1-s_{2}+s_{4}\right)=0 \\
\therefore \quad \alpha+\beta+\gamma+\delta=n \pi, \text { and not }(2 n+1) \pi \text { necessarily. }
\end{gathered}
$$


Let us now denote $\tan \frac{\phi}{2}$ by $t$, when we get

$$
\begin{aligned}
& a \cdot \frac{1+t^{2}}{1-t^{2}}-b \cdot \frac{1+t^{2}}{2 t}=c \\
\therefore \quad & b\left(t^{4}-1\right)+2(a+c) t^{3}+2(a-c) t=0,
\end{aligned}
$$

where $t^{2}$ is absent, and $s_{4}=-1$, so that $1-s_{2}+s_{4}=0$ while $s_{1}-s_{3}$ is not $=0$. Thus

$$
\begin{aligned}
\tan \frac{1}{2}(\alpha+\beta+\gamma+\delta) & =\infty \\
\therefore \quad \frac{1}{2}(a+\beta+\gamma+\delta) & =n \pi+\frac{\pi}{2} \\
a+\beta+\gamma+\delta & =(2 n+1) \pi,
\end{aligned}
$$

and

which is the correct result.

The reason why the first biquadratic does not give the exact result being that, to obtain it, we performed the operation of squaring; and when we square we must expect, as usual, a result of greater generality than, but not contradictory of, the actual result. 